\title{
The association of endothelial cell signaling, severity of illness, and organ dysfunction in sepsis
}

\author{
Nathan I Shapiro ${ }^{1,2^{*}}$, Philipp Schuetz ${ }^{1}$, Kiichiro Yano ${ }^{1,2}$, Midori Sorasaki ${ }^{1,2}$, Samir M Parikh ${ }^{3}$, Alan E Jones ${ }^{5}$, \\ Stephen Trzeciak ${ }^{6}$, Long $\mathrm{Ngo}^{4}$, William C Aird ${ }^{2}$
}

\begin{abstract}
Introduction: Previous reports suggest that endothelial activation is an important process in sepsis pathogenesis. We investigated the association between biomarkers of endothelial cell activation and sepsis severity, organ dysfunction sequential organ failure assessment (SOFA) score, and death.

Methods: This is a prospective, observational study including adult patients (age 18 years or older) presenting with clinical suspicion of infection to the emergency department (ED) of an urban, academic medical center between February 2005 and November 2008. Blood was sampled during the ED visit and biomarkers of endothelial cell activation, namely soluble fms-like tyrosine kinase-1 (sFlt-1), plasminogen activator inhibitors -1 (PAI-1), sE-selectin, soluble intercellular adhesion molecule (sICAM-1), and soluble vascular cell adhesion molecule (sVCAM-1), were assayed. The association between biomarkers and the outcomes of sepsis severity, organ dysfunction, and inhospital mortality were analyzed.
\end{abstract}

Results: A total of 221 patients were included: sepsis without organ dysfunction was present in $32 \%$, severe sepsis without shock in 30\%, septic shock in 32\%, and $6 \%$ were non-infected control ED patients. There was a relationship between all target biomarkers (sFlt-1, PAl-1, sE-selectin, sICAM-1, and sVCAM-1) and sepsis severity, $P<0.05$. We found a significant inter-correlation between all biomarkers, including the strongest correlations between sFlt-1 and sE-selectin $(r=0.55, P<0.001)$, and between sFlt-1 and PAl-1 $(0.56, P<0.001)$. Among the endothelial cell activation biomarkers, sFlt-1 had the strongest association with SOFA score $(r=0.66, P<0.001)$, the highest area under the receiver operator characteristic curve for severe sepsis of 0.82 , and for mortality of 0.91 .

Conclusions: Markers of endothelial cell activation are associated with sepsis severity, organ dysfunction and mortality. An improved understanding of endothelial response and associated biomarkers may lead to strategies to more accurately predict outcome and develop novel endothelium-directed therapies in sepsis.

\section{Introduction}

Despite recent advances in biomedical research, sepsis remains an important medical challenge. An estimated 750,000 cases of severe sepsis are diagnosed each year in the United States alone [1], incurring health care costs of $\$ 16.7$ billion annually [2]. One major potential shortcoming of prior therapeutic approaches in sepsis is the attempt to target one specific pathway, component, or

\footnotetext{
* Correspondence: nshapiro@bidmc.harvard.edu

'Department of Emergency Medicine, Beth Israel Deaconess Medical

Center,1 Deaconess Road CC2-W, Boston, MA 02215, USA Full list of author information is available at the end of the article
}

cytokine involved in the host response; however, the host response in sepsis is coordinated across multiple pathways including inflammation, coagulation, metabolism and tissue hypoxia. An important goal in sepsis research is to develop a more detailed understanding of the mechanisms underlying the host response to infection, with the expectation that such studies will yield novel insights into potential diagnostic and therapeutic targets.

There is increasing evidence that the endothelium plays a central and pathogenic role in sepsis. Endothelial cells are diverse in function and highly responsive to
Ciomed Central 
their extracellular environment (reviewed in [3]). When exposed to certain agonists, such as lipopolysaccharide, cytokines, chemokines or growth factors, endothelial cells become activated. The activation state is manifested by enhanced permeability, increased leukocyte adhesion, a shift in the hemostatic balance towards a procoagulant phenotype, and altered regulation of vasomotor tone. Collectively, these changes likely evolved as an adaptive host response to extravascular pathogens, allowing for increased blood flow to the area of insult, local efflux of plasma proteins and leukocytes, and sequestering of the infection. This activated state may be considered dysfunctional when an overactive endothelium disturbs the homeostatic state instead of restoring it, representing a net liability to the host. In this context, endothelial dysfunction typically involves some combination of increased leukocyte adhesion and transmigration, increased permeability, a shift in the hemostatic balance towards the procoagulant side and an alteration in vasomotor tone. In sepsis, endothelial activation and dysfunction are critical determinants of the host response and, thus, represent a unifying explanation for the complex sepsis pathophysiology, as well as an attractive target for systemic therapy.

The aim of the present study was to assay a broad range of endothelial markers in a large sample of human patients at the time of emergency department (ED) presentation with the goal of gaining further insights into the activation state of the endothelium in different stages of sepsis. To that end, we have measured circulating levels of soluble leukocyte adhesion molecules (soluble vascular cell adhesion molecule (VCAM)-1, soluble intercellular adhesion molecule (ICAM-1) and sE-selectin; procoagulant/antifibrinolytic mediators (plasminogen activator inhibitors (PAI)-1); and a marker of vascular endothelial growth factor (VEGF) signaling (sFlt-1) (reviewed in Figure 1) in 221 septic patients with varying degrees of severity. We analyzed the relationships between the biomarkers of endothelial cell activation and sepsis severity, inflammatory response, organ dysfunction, and mortality. An improved understanding of the endothelial cell response in sepsis may suggest avenues for diagnostic platforms, and could also delineate new strategies for identifying patients with endothelial cell dysfunction that may be particularly responsive to therapies targeted to restore endothelial health.

\section{Materials and methods}

\section{Design and population}

This was a prospective, cohort study of a convenience sample of adult patients (age 18 years or older) presenting to the ED with suspected infection. Suspected infection was defined as a clinical suspicion of an infectious etiology as assessed by the treating clinician, and determined by interviewing the treating physician to determine if infection was suspected based on the ED work-up including the results from history, physical exam, laboratory and diagnostic testing. The population was selectively enrolled to achieve a relatively even distribution of different sepsis severities. A sample of non-infected ED control patients was also assembled by identifying adult ED patients without evidence of infection during presentation. The study period was between February 2005 and November 2008. There were 221 patients enrolled in the study with 189 patients enrolled de novo, and 32 patients co-enrolled with another protocol [4]. The setting was Beth Israel Deaconess Medical Center (BIDMC), Boston, an urban teaching hospital. The study was approved by the hospital ethics board, and written informed consent was obtained.

\section{Collection of clinical covariates}

In order to characterize the population, relevant components of demographics, history, co-morbid diseases, suspected source of infection, vital sign information, physical exam findings, and the results of laboratory and radiologic testing were collected. The Charlson comorbidity index, a well established methodology to quantify co-morbid disease burden, was calculated for each patient [5].

\section{Biomarker analysis}

All subjects received a blood draw while in the emergency department. Samples were drawn in EDTA tubes, centrifuged at $2,500 \times \mathrm{g}$ at $4^{\circ} \mathrm{C}$, and frozen at $-80^{\circ} \mathrm{C}$ within one hour of collection. Plasma was assayed for sE-selectin, sICAM-1, sVCAM-1, and PAI-1 as a multiplex panel using the human cardiovascular-1 panel (Millipore, Billerica, MA, USA) and Interleukin-6 (IL-6) using the human cardiovascular-3 panel (Millipore) on the Luminex 200 instrument (Millipore). The sFlt-1 assays were performed using Quantikine ELISA kits (R\&D systems, Minneapolis, MN, USA). All assays were performed in duplicate and the average levels were used for analysis.

\section{Septic shock subset with daily blood draws}

Between January 2007 and January 2009, patients in our study with septic shock received additional blood draws every 24 hours for the first three days - a total of 52 patients were enrolled in this subset. This sub-study was performed to assess the changes in the circulating biomarkers of endothelial cell activation over time.

\section{Outcomes assessment}

\section{Sepsis severity classification}

Sepsis severity was characterized according to a modified version of the ACCP/SCCM sepsis syndromes [6]. 


\section{EC Response to Sepsis}

\section{Leukocyte trafficking (Inflammation)}

(a)

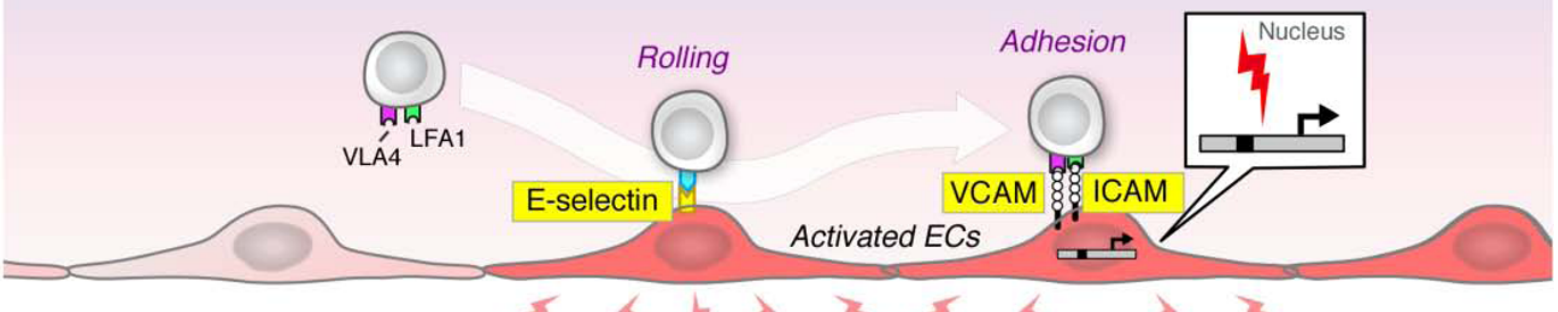

(b)

\section{Coagulation activity (Inhibition of fibrinolysis)}

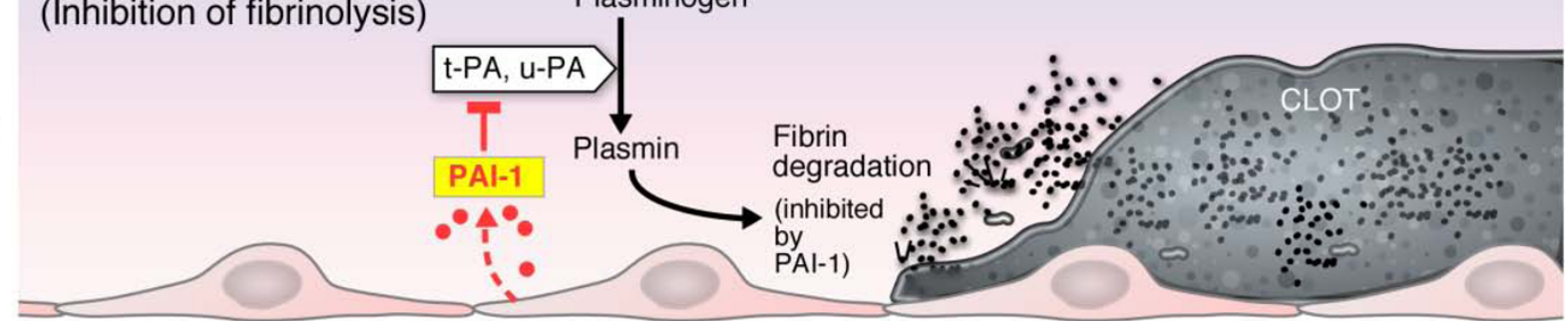

\section{Plasminogen}

\section{VEGF signalling} Quiescence

(c)
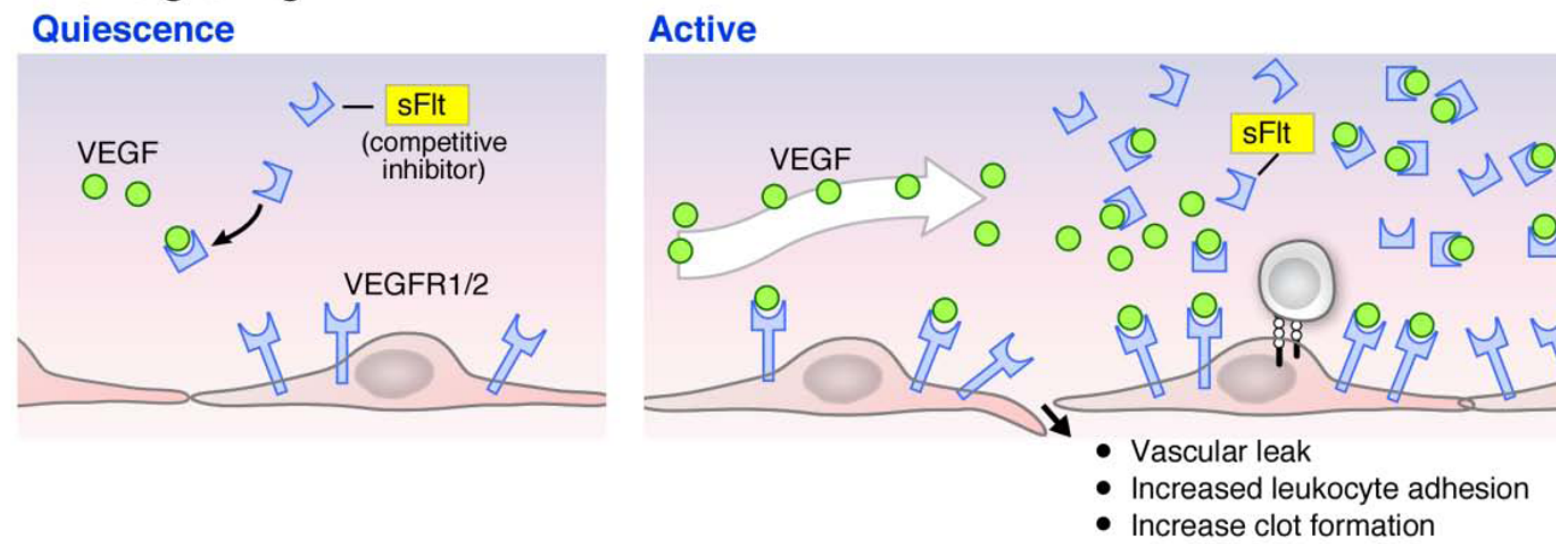

Figure 1 Endothelial cell response in sepsis. (a) Leukocyte trafficking. Activated endothelial cells (red-colored cells) express increased levels of E-selectin, P-selectin, intercellular adhesion molecule (ICAM)-1 and vascular cell adhesion molecule (VCAM)-1 (all but P-selectin are shown). Upregulation of E-selectin, ICAM-1 and VCAM-1 are mediated at a transcriptional level (activation signal and promoter with transcriptional start site are shown in the inset). E selectin induces rolling of circulating leukocytes. VCAM-1 and ICAM-1 induce firm adhesion of leukocytes by binding to very late antigen 4 (VLA4) and leukocyte function antigen LFA1, respectively. Following firm adhesion, leukocytes transmigrate through and/or between endothelial cells into the underlying tissue (not shown). In sepsis, E-selectin, ICAM-1, and VCAM-1 are cleaved from the cell surface and circulate as a soluble form of the receptor. Circulating levels are indirect measures of the degree of endothelial activation. (b) Hemostasis. Activated endothelial cells undergo a net shift in hemostatic balance towards the procoagulant side, leading to local clot formation. During fibrinolysis tissue-type plasminogen activator ( $t-P A)$ and urokinase-type plasminogen activator (u-PA) mediate the conversion of plasminogen to plasmin. Plasmin, in turn, proteolytically degrades fibrin. Activated endothelial cells express increased levels of plasminogen activator inhibitor (PAl-1), which inhibit the activity of t-PA and u-PA, thus accentuating the procoagulant state. (c) Vascular endothelial growth factor (VEGF) signaling. Under normal conditions (quiescence), VEGF signaling plays a critical role in homeostasis. VEGF binds to two receptors on endothelial cells, VEGF receptor (VEGFR) 1 and 2. VEGFR1 is also known as Flt-1. In sepsis (activated state), circulating levels of VEGF are increased. Elevated VEGF signaling, in turn, leads to increased vascular leak, leukocyte adhesion/trafficking, and clot formation. Sepsis is also associated with increased circulating levels of a soluble form of VEGFR1 (sFlt-1). sFlt-1 binds VEGF in the blood, thus acting as a competitive inhibitor of VEGF signaling in endothelial cells. Sepsis-mediated induction of sFlt-1 may represent a critical component of the host anti-inflammatory response. 
We have previously published on the details and validity of these modified definitions [7]. Patients were characterized into one of the following groups: non-infected ED patients, sepsis, severe sepsis, or septic shock. For assessment of organ dysfunction, we used the SOFA score, and for additional severity of illness assessment [8], we used the Acute Physiologic And Chronic Health Evaluation (APACHE)-II score [9] based on the worst values over the first 24 hours, as originally described. Serum lactate levels were used as another severity of illness marker [10] and were either obtained as part of routine clinical care, or assayed using a point-of-care istat device (Abbott Point-of-Care, Princeton, NJ, USA). We have previously affirmed the concordance of these two methods [11].

\section{Sepsis severity classification}

Non-infected ED patients were defined as patients presenting to the ED without a clinical suspicion of infection. Sepsis was comprised ED patients with suspected infection with or without systemic inflammatory response syndrome (SIRS). The decision to combine these groups (with and without SIRS) was based on our previous publication demonstrating no mortality difference based on SIRS criteria alone so that severity is equivalent $[7,12]$. Severe Sepsis was defined as sepsis with concomitant organ dysfunction defined by meeting one or more of the following organ dysfunction definitions; central nervous system: new altered mental state and/or new onset of GCS $<15$; respiratory: any mechanical ventilation, supplemental oxygen required to maintain oxygen saturation $>95 \%$, and/or respiratory rate $>24$ beats per minute; cardiovascular: any vasopressor use, SBP < $90 \mathrm{mmHg}$ after $20 \mathrm{~mL} / \mathrm{kg}$ bolus; renal: urine output $<0.5$ $\mathrm{mL} / \mathrm{kg} / \mathrm{hr}$, or creatinine $>50 \%$ of baseline or $>2 \mathrm{mg} / \mathrm{dl}$ if baseline is unknown; hepatic: AST/ALT > 80 (new); hematopoietic: platelet count $<100,000$ and/or PT/PTT $>50 \%$ of normal; or metabolic: lactate $>2.5 \mathrm{mmol} / \mathrm{l}$. Septic shock was defined as sepsis plus hypotension (SBP < $90 \mathrm{mmHg}$ after 20 to $30 \mathrm{cc} / \mathrm{kg}$ fluid challenge). The sepsis severity was assessed on presentation and daily for the first 72 hours or until hospital discharge, assigning a patient to the worst syndrome achieved on a daily basis.

\section{Organ dysfunction}

The sequential organ failure assessment (SOFA) score was used to assess organ failures [8]. The SOFA score is designed to identify morbidity and individualizes the dysfunction or failure of each organ system. It has been established as a valid predictor for both initial and serial assessments [13-15]. The SOFA score was assessed on presentation and then daily for the first 72 hours or until hospital discharge.

\section{Other Inflammatory response and IIIness severity markers}

IL-6 level was used as a prototype marker of inflammatory response. APACHE-II score was used as a secondary assessment of severity based on worst vital signs, as originally described [9]. This score has been validated as an assessment tool for risk-stratification, and was utilized to characterize disease severity. While some of the baseline variables make it a score that is not necessarily responsive to acute disease state, its prognostic ability has been well established. The APACHE-II score was assessed on presentation, and then daily for the first 72 hours or until hospital discharge. Mortality was defined by hospital discharge disposition.

\section{Statistical analysis}

Means with standard deviations, medians with interquartile ranges, and proportions were used for descriptive statistics, as appropriate. To analyze the association between the biomarkers of endothelial cell activation and sepsis severity, we used generalized linear modeling. Next, we calculated Spearman rank correlation coefficients to assess the bivariable association among the biomarkers. We display the graphs with a regression line and reported the calculated Spearman correlation coefficient ( $r$-value) along with the associated $P$-value. We performed a similar analysis between the target biomarkers and organ dysfunction (SOFA score), the inflammatory response marker IL-6, and APACHE-II score. Due to non-normal distribution, SOFA score was log transformed throughout the analysis. As a comparator, we also examined the correlation of IL-6 and serum lactate with SOFA score. Next, to compare the strength of association between each of the biomarkers and organ dysfunction, we standardized each of the biomarkers values through the following formula: ((biomarker - biomarker mean)/ biomarker SD). We then used a linear regression model and adjusted for age, gender, and co-morbid illness burden (Charlson score). We report the beta coefficient with standard error as well as the adjusted $r$-squared value for each biomarker model. We also tested multi-marker models to determine the value of combinations of biomarkers. To assess the clinical predictive ability of the biomarkers, we calculated the area under the receiver operating characteristic curve (AUC) with 95\% confidence interval for each biomarker to predict the outcomes of severe sepsis (including septic shock) within 72 hours and in-hospital mortality. The AUCs were compared nonparametric approach [16].

Finally, for the subset analysis of biomarkers from patients with septic shock collected daily over the first 72 hours of hospitalization, we used a linear mixed effects model to estimate the differences in biomarkers between survivors and non-survivors over time. The linear mixed-effects model took into account the multiple measurements (at 0, 24, 48, 72 hours) of biomarkers 
and outcomes and used compound symmetry variancecovariance structure to account for the within-subject correlation.

\section{Results}

Population characteristics

There were a total of 221 patients enrolled with a mean age of 58 (SD +/ - 19) years; $52 \%$ were male, $76 \%$ Caucasian, and there was a high co-morbid burden: diabetes $(26 \%)$, cancer $(20 \%)$ and chronic heart failure in $13 \%$ (Table 1). On admission, sepsis without organ dysfunction was present in $32 \%$, severe sepsis without shock in $30 \%$, and septic shock in $32 \%$. Six percent were noninfected ED patients who were used as controls. The overall in-hospital mortality in the population was $7.7 \%$ $(13 / 221)$, and $42 \%(84 / 221)$ of patients were admitted to the intensive care unit (ICU).

\section{Table 1 Patient characteristics}

\begin{tabular}{|c|c|}
\hline Parameters & $\begin{array}{l}\text { Overall } \\
n=221\end{array}$ \\
\hline \multicolumn{2}{|l|}{ Demographics } \\
\hline Age median, mean (SD) & $57,58(19)$ \\
\hline Race: white n (\%) & $169(76 \%)$ \\
\hline african-american & $28(13 \%)$ \\
\hline Other & $24(11 \%)$ \\
\hline Female gender n (\%) & 115 (52\%) \\
\hline \multicolumn{2}{|l|}{ Comorbidities n (\%) } \\
\hline COPD & $16(7 \%)$ \\
\hline Chronic Heart failure & $29(13 \%)$ \\
\hline Diabetes & $63(28 \%)$ \\
\hline Cancer & $45(20 \%)$ \\
\hline \multicolumn{2}{|l|}{ Sepsis Syndrome n(\%) } \\
\hline Non-infected ED patients & $14(6 \%)$ \\
\hline Sepsis without organ dysfunction & $70(32 \%)$ \\
\hline Severe Sepsis without shock & $66(30 \%)$ \\
\hline Septic Shock & $71(32 \%)$ \\
\hline \multicolumn{2}{|c|}{ Severity of Disease, median, mean (SD) } \\
\hline SOFA score & $2,3(4)$ \\
\hline APACHE score & $11,12(8)$ \\
\hline Lactate (mg/dL) & $1.5,2.1(1.7)$ \\
\hline \multicolumn{2}{|l|}{$\begin{array}{l}\text { Marker levels on admission* } \\
\text { median, mean (SD) }\end{array}$} \\
\hline Eselectin $(\mathrm{ng} / \mathrm{mL})$ & $49.3,67.5(55.4)$ \\
\hline VCAM-1 (ng/mL) & $1,120,1,411(1,316)$ \\
\hline ICAM-1 (ng/mL) & $176,224(151)$ \\
\hline PAl-1 (ng/mL) & $40.964 .6(644)$ \\
\hline sFlt-1 (pg/mL) & $118,194(224)$ \\
\hline
\end{tabular}

\section{Endothelial cell activation and sepsis severity}

We found an association between biomarker levels and sepsis severity (worst sepsis syndrome within 72 hours) for sFlt-1 $(P<0.001$ for trend across groups), PAI-1 $(P<0.001)$, sE-selectin $(P<0.001)$, sICAM-1 $(P<0.05)$, and sVCAM-1 $(P<0.04)$ (Figure 2$)$. The most significant increases were found in median sFlt-1 levels, which ranged from $41 \mathrm{ng} / \mathrm{ml}$ (IQR 31 to 51) in non-infected controls to $243 \mathrm{ng} / \mathrm{ml}$ (IQR 137 to 449 ) in septic shock; and, in PAI-1 which ranged from $25.3 \mathrm{ng} / \mathrm{ml}$ (IQR 17.6 to 36.8 ) to $76.7 \mathrm{ng} / \mathrm{ml}$ (IQR 49.4 to 136 ).

\section{Evidence of endothelial cell activation}

To assess whether there was evidence of endothelial cell activation in the response to infection, we correlated the selected biomarkers which individually represent various components of the endothelial cell signaling pathway. Using a Spearman rank correlation coefficient, we found a significant correlation between all biomarkers (sFlt-1, PAI-1, sE-selectin, sICAM-1, and sVCAM-1) (Figure 3). The strongest correlations were between sFlt-1 and sEselectin $(\mathrm{r}=0.55, P<0.001)$ and sFlt-1 and PAI- $1(0.56$, $P<0.001)$.

\section{Endothelial cell activation biomarkers and organ dysfunction}

To assess the association of endothelial cell related biomarkers with organ dysfunction, we analyzed the correlation between the endothelial related biomarkers with SOFA score in the ED. All biomarkers were significantly correlated with the concurrent SOFA score (Figure 4). Of note, sFlt- 1 was highly correlated $(r=0.66, P<$ 0.001 ) with SOFA score, and compared favorably in predicting SOFA score to other common biomarkers of inflammation such as IL-6 $(\mathrm{r}=0.45)$ and lactate $(\mathrm{r}=$ 0.43 ). In addition, biomarker levels at the time of presentation correlated with SOFA score at 24 hours: sEselectin (0.37), sFlt-1 (0.64), sVCAM-1 (0.22), and PAI-1 (0.51), $P<0.001$ for all comparisons; except sICAM-1 (0.13), $P=0.08$.

\section{Endothelial cell activation biomarkers and inflammation}

We used circulating IL- 6 concentrations as a read-out of the pro-inflammatory response. There was a notable association between the biomarker levels of endothelial activation and IL-6 (Figure 4). Here, sFlt-1 had a particularly strong correlation with IL-6 $(\mathrm{r}=0.62, P<0.001)$.

\section{Endothelial cell activation biomarkers and other severity of illness markers}

Endothelial cell activation markers correlated with two independent markers of disease severity, lactate and APACHE-II scores. There was a significant correlation using Spearman rank between the target biomarkers and 


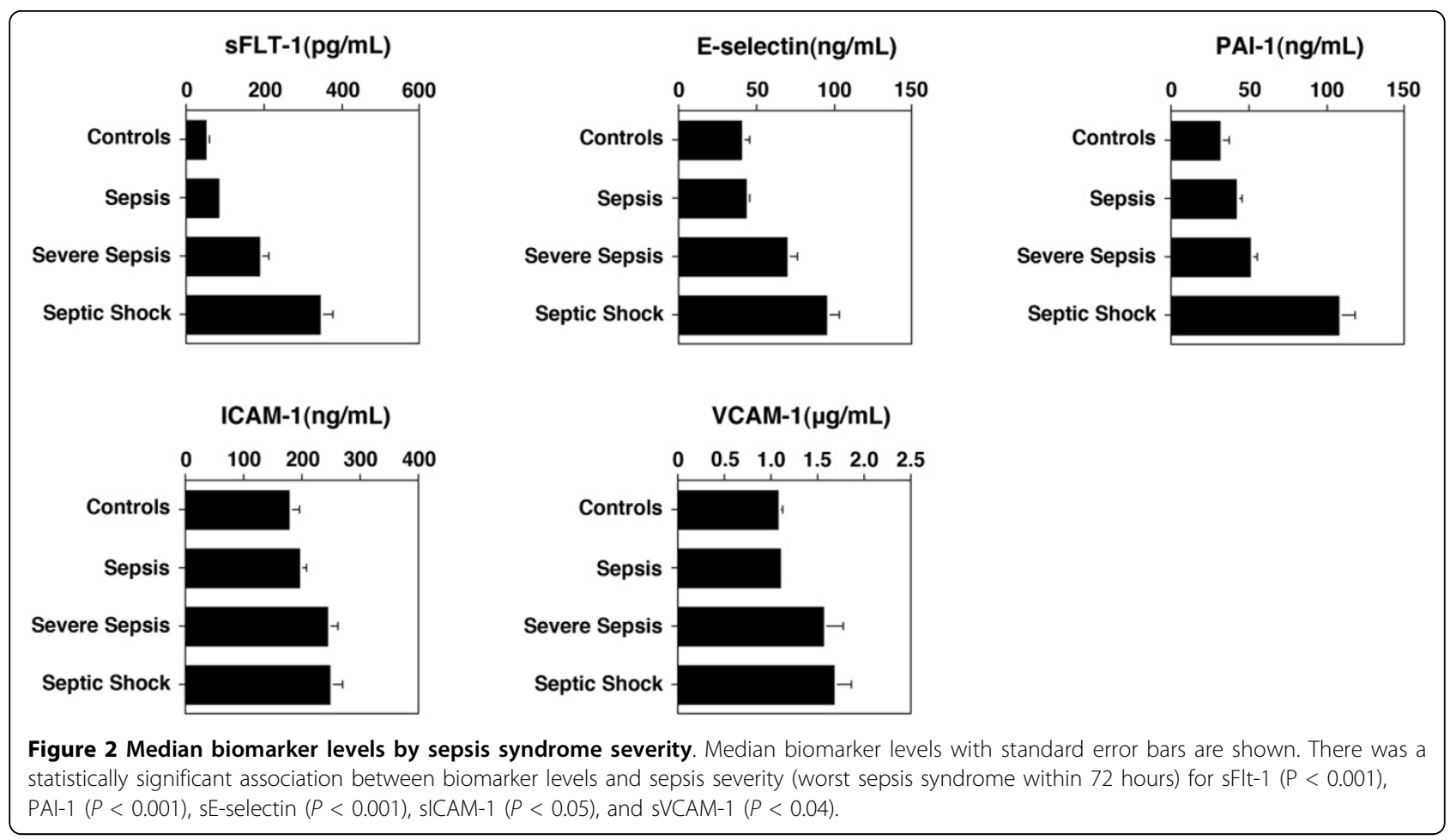

APACHE-II score: sFlt- $1(\mathrm{r}=0.58, P<0.01)$, pai1 $(0.46$, $P<0.01)$, sE-selectin $(0.33, P<0.01)$, sICAM-1 $(0.15$, $P<0.03)$, and sVCAM-1 $(0.25, P<0.01)$. These results compare favorably to the $r$-value for the correlation between classic biomarkers such as lactate with APACHE-II (0.38) and IL-6 with APACHE-II (0.43). There was a significant association between the endothelial related biomarkers and lactate level: sFlt-1 (0.51, $P<0.01)$, PAI-1 $(0.40, P<0.01)$, sE-selectin $(0.33$, $P<0.01)$, sICAM-1 $(0.23, P<0.01)$, and sVCAM-1 (0.20, $P<0.01)$. As a comparator, IL-6 correlation coefficient with lactate was 0.44 .

\section{Biomarker association with organ dysfunction adjusted} for age, gender, and co-morbid illness burden

We analyzed the association of the biomarkers with organ dysfunction (log SOFA score) with linear regression models adjusted for age, gender, and co-morbid illness burden (Table 2) using beta coefficients standardized to a 0 to 100 scale to allow equal comparison. We report the models testing one marker at a time (Table 2). We then checked to see if model fit (measured by adjusted $\mathrm{R}^{2}$ ) would be improved by any combination of multiple markers in the model. Interestingly, once sFlt-1 was included in the models, no additional marker becomes significant if added. The $R^{2}$ value in the adjusted model for sFlt- 1 alone was 0.46 , and adding any second marker did not improve the model fit above this level. Additionally, and there was no other combination of two or more markers that exceeds the $\mathrm{R}^{2}$ of the model with sFlt- 1 alone, including adding IL- 6 and lactate as eligible covariates. Thus, the marker sFlt1 appears to have the strongest association with organ dysfunction.

Biomarkers as predictors of severe sepsis and mortality To further assess the clinical accuracy of the different markers, we report the area under the receiver operating characteristic curve for the ability of the biomarker drawn on ED presentation to predict two clinical outcomes: 1) severe sepsis (including septic shock as cardiovascular dysfunction) within 72 hours; and, 2) inhospital mortality (Table 3). Again, sFlt-1 performed with the highest accuracy, and has a higher AUC (0.82; 95\% CI 0.76 to 0.88 ) for severe sepsis when compared to all other endothelial related biomarkers $(P<0.05)$. For the outcome of in-hospital mortality, sFlt-1 had an AUC of 0.91 (0.87 to 0.95$)$, and was also higher $(P<$ 0.05 ) than the AUC for all other markers (Table 3).

\section{Performance of daily markers in septic shock}

There were a total of 52 patients with septic shock who in addition to the 0 hour draw had serial samples at 24, 48 and 72 hours. We compared biomarker levels in survivors $(n=43)$ to non-survivors $(n=9)$ (Figure 5). Using a linear mixed-effects model, adjusting for age, gender, and co-morbid burden, we found the following estimated mean differences in biomarker levels over 


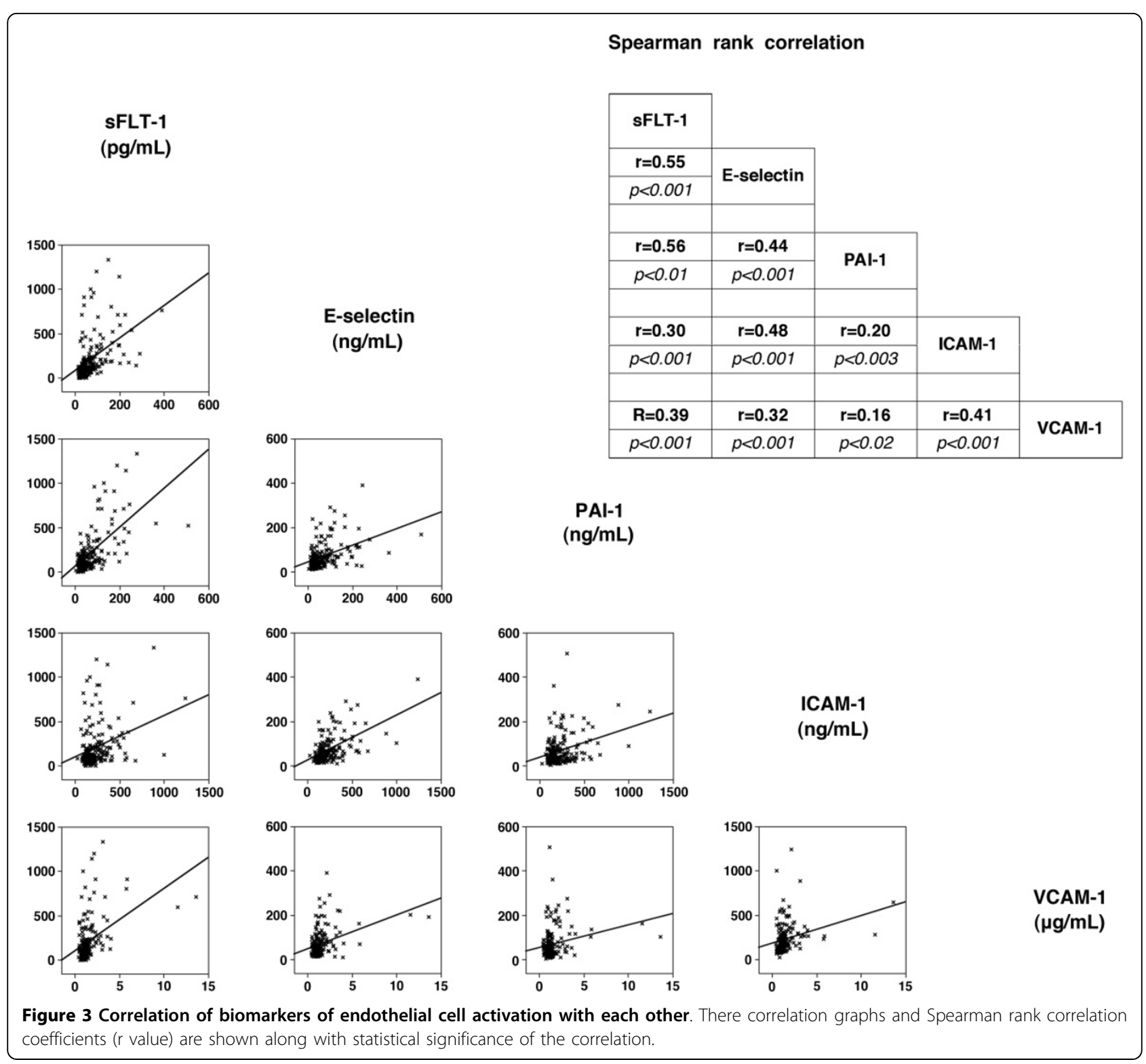

time comparing the non-survivors to survivors: sFlt-1 $366 \mathrm{pg} / \mathrm{mL}$ (95\% CI: 218 to 514, $P<0.01$ ); PAI-1 63.2 $\mathrm{ng} / \mathrm{ml}(38.5$ to $87.8, P<0.01)$; sE-selectin $24.1 \mathrm{ng} / \mathrm{mL}$ (5.5 to $42.7, P<0.01$ ); sICAM-1 $135 \mathrm{ng} / \mathrm{mL}$ (67 to 202 , $P<0.01)$; and, sVCAM-1 $683 \mathrm{ng} / \mathrm{mL}$ (320 to $1,046, P<$ 0.01).

\section{Discussion}

The endothelium plays a key role in mediating vasomotor tone, leukocyte trafficking, permeability, and hemostasis (reviewed in [17,18]; Figure 1). Activation and dysfunction of the endothelium is characterized by increased permeability, vasodilation, recruitment of leukocytes, and a shift in the hemostatic balance towards the procoagulant side. Our findings in a group of moderately ill emergency department patients (mortality rate $=8 \%, 40 \%$ ICU admission rate) that sepsis severity is associated with increased circulating levels of sFlt-1, sICAM-1, sVCAM-1, sE-selectin and PAI-1 are consistent with the hypothesis that the endothelium is activated in sepsis.

Leukocyte trafficking across the endothelium involves a tightly regulated multistep process (reviewed in [19], Figure 1). Endothelial E-selectin and P-selectin regulate leukocyte rolling on the endothelium, whereas ICAM-1 and VCAM-1 are involved in firm adhesion. Many in vitro studies have demonstrated that activation agonists induce the mRNA and protein expression of these cell adhesion molecules. Expression levels are also increased in animal models of sepsis [20,21]. In contrast 

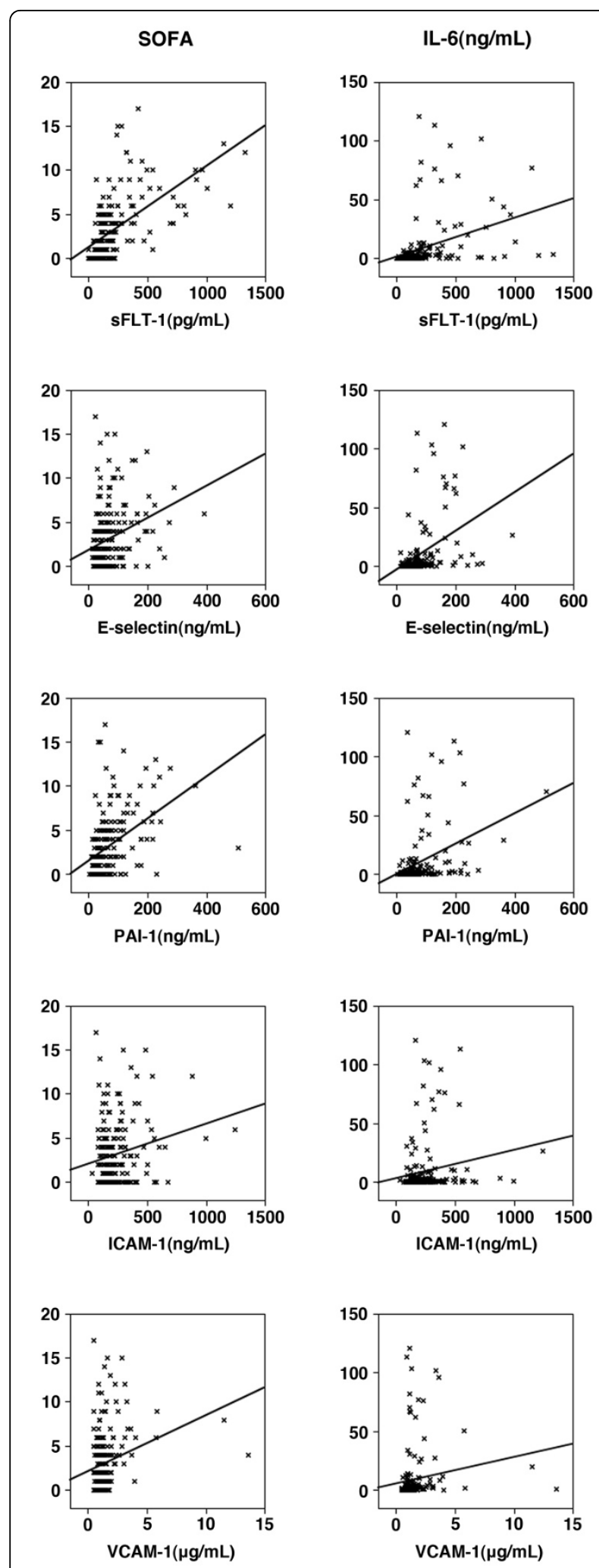

Figure 4 Correlation of biomarkers of endothelial cell activation with SOFA score and IL-6. The correlation graphs and Spearman rank correlation coefficients ( $r$ value) are shown along with statistical significance of the correlations.
Table 2 Association of individual biomarkers with organ dysfunction, adjusted for age, gender, and comorbid burden

\begin{tabular}{lcccc}
\hline & \multicolumn{4}{c}{$\begin{array}{c}\text { Organ dysfunction } \\
\text { (log tranformed SOFA score) }\end{array}$} \\
\cline { 2 - 5 } Biomarker & Std. beta & SE & P-value & Model adj. $\mathbf{r}^{\mathbf{2}}$ \\
\hline sFlt-1 & 0.39 & 0.05 & $<0.001$ & 0.46 \\
PAI-1 & 0.29 & 0.05 & $<0.001$ & 0.38 \\
E-selectin & 0.20 & 0.05 & $<0.001$ & 0.33 \\
ICAM-1 & 0.11 & 0.05 & $<0.04$ & 0.29 \\
VCAM-1 & 0.15 & 0.05 & $<0.003$ & 0.30 \\
\hline
\end{tabular}

Table 2 shows the results from each individual biomarker incorporated into a linear regression model (one marker per model) with outcome SOFA, adjusted for age (years), gender, and co-morbid burden (charlson index). Thus, each line represents its own model: Expected log SOFA $=$ intercept $+\alpha$ (Biomarker) $+\beta$ (Age) $+\chi$ (gender) $+\delta$ (Charlson). The biomarkers are standardized [(biomarker - biomarker mean)/SD] so the beta estimates are comparable. Each biomarker showed a statistically significant association with SOFA score. sFlt-1 demonstrates the largest beta estimate which is also supported by an adjusted $r$-squared in the model of 0.46 .

to animal models, there are currently no reliable assays for adhesion molecules in the intact endothelium of humans. In a recent proof-of-concept study, we showed the potential value of skin biopsies for assaying adhesion molecule expression in sepsis [21]. However, the protocol is invasive, and the data do not necessarily extrapolate to vascular beds outside the skin. A more common approach is to measure circulating levels of soluble adhesion molecule receptors as surrogate markers of endothelial activation. P- and E-selectin, ICAM-1 and VCAM-1 all undergo proteolytic cleavage of the extracellular region of the membrane-bound receptor [22-25] and levels of these soluble forms are increased in experimental and clinical sepsis [26-34]. Consistent with these published reports, our results show that sepsis is associated with elevated circulating levels of soluble ICAM1, VCAM-1 and E-selectin. The levels were directly correlated with severity of illness and SOFA score, supporting the notion that the endothelium undergoes graded activation during the host response to infection.

Table 3 Area under the curve for each biomarker as a predictor of severe sepsis and death

\begin{tabular}{lllll}
\hline & \multicolumn{4}{c}{ Outcome } \\
\cline { 2 - 5 } Biomarker & AUC & $95 \% \mathrm{Cl}$ & AUC & $\mathbf{9 5 \%} \mathrm{Cl}$ \\
\cline { 2 - 5 } sFIt-1 & $0.82^{*}$ & 0.76 to 0.88 & $0.91^{*}$ & 0.87 to 0.95 \\
PAI-1 & 0.69 & 0.62 to 0.76 & 0.74 & 0.60 to 0.88 \\
Eselectin & 0.71 & 0.64 to 0.78 & 0.65 & 0.49 to 0.82 \\
Icam & 0.61 & 0.53 to 0.69 & 0.72 & 0.57 to 0.87 \\
Vcam & 0.60 & 0.52 to 0.69 & 0.57 & 0.35 to 0.79 \\
\hline
\end{tabular}

*the area under the curve for sFlt-1 in predicting both severe sepsis (includes patients with septic shock) and mortality was significantly greater than all other AUC values, $P<0.01$ 


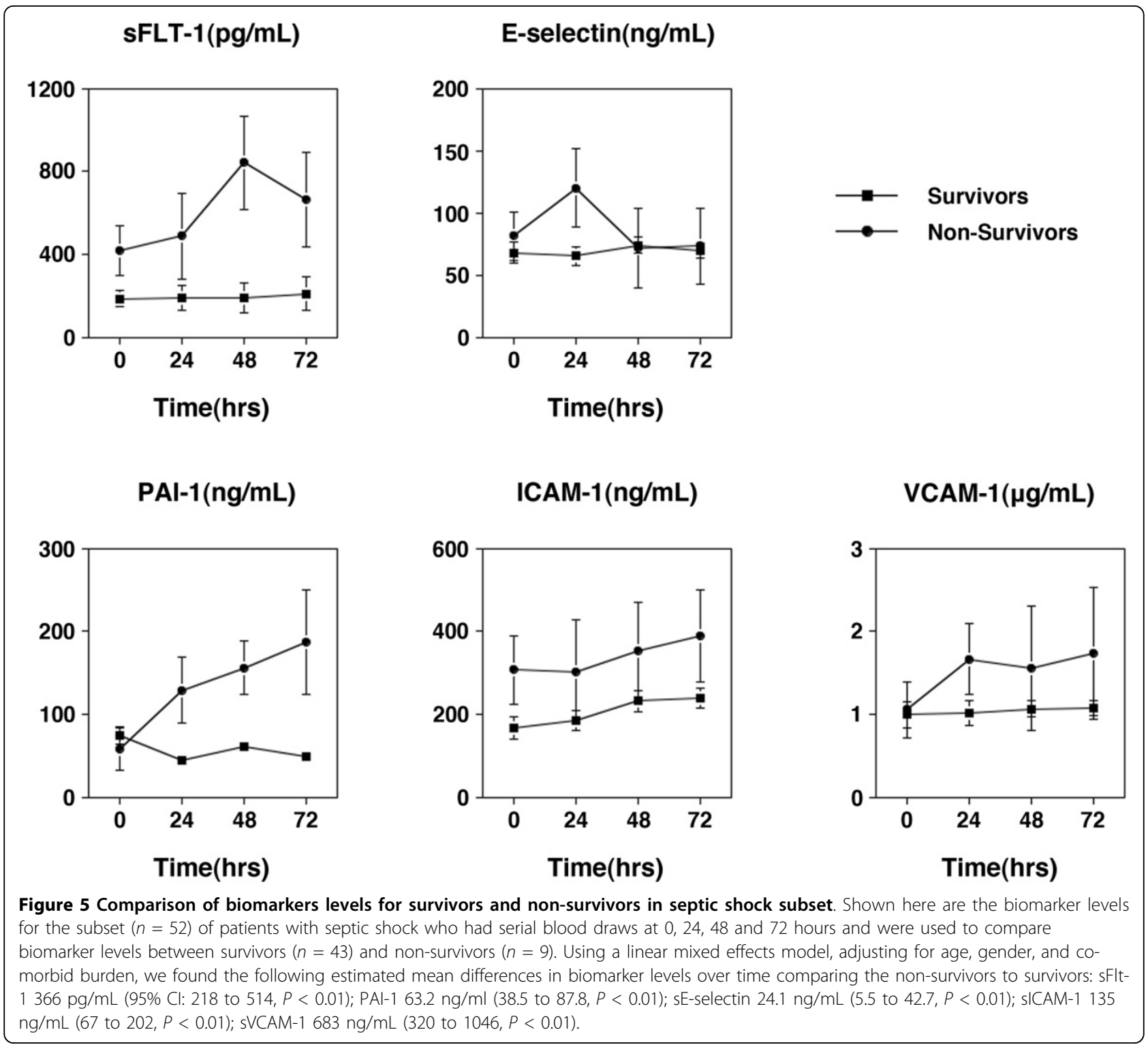

The endothelium also balances hemostasis, which too, is deranged in sepsis (reviewed in [35]). Consistent with the results of previous studies [36-41], we have shown that PAI-1 levels are increased in severe sepsis, and that such levels correlate with the degree of severity. Since PAI-1 is largely restricted in its expression to endothelial cells, these findings add further support to the conclusion that the endothelium becomes increasingly activated during the host response.

Using animal models of sepsis, we have recently shown that VEGF plays an important role in mediating sepsis pathophysiology [20]. The biological plausibility of these findings is supported by the observation that VEGF signaling in endothelial cells results in an activation phenotype, including increased permeability, induction of cell adhesion molecules [42-44], the release of cytokines and chemokines, and the expression of procoagulant molecules [44]. VEGF binds to two receptors on the surface of endothelial cells, Flk-1 (also known as VEGFR2 or KDR) and Flt-1 (also known as VEGFR1). Flt-1 is also produced as a soluble receptor, sFlt-1, via alternative splicing of the precursor mRNA and functions as a decoy molecule, competing with membranebound Flt-1 for binding to VEGF. Indeed, we showed that the systemic administration of sFlt-1 (levels of approximately 20 -fold over baseline) blocked sepsis morbidity and mortality in mice. Interestingly, endotoxin challenge in mice resulted in elevated (approximately five-fold) circulating levels of sFlt-1. We confirmed these observations in a small number of 
human patients with severe sepsis [4]. Together, these data suggested that sFlt-1 contributes to the systemic anti-inflammatory host response to infection. In the current study, we have extended these findings by showing that sFlt-1 is increased in patients with sepsis and that it is a superior marker of sepsis severity compared with the other markers tested.

Our findings add to the existing literature in important ways. First, with the exception of a study in which PAI-1 levels were measured in 840 patients with severe sepsis enrolled in the PROWESS trial [36], the current report includes the largest cohort of sepsis patients analyzed to date for soluble markers of endothelial activation. Second, the study is the only one that we are aware of that has included endothelial markers of both leukocyte adhesion and coagulation in the same population of patients. The finding that sFlt-1 levels correlate more closely with severity of illness and are a stronger predictor of organ dysfunction and mortality compared with soluble adhesion molecule receptors, IL-6, and lactate is novel. Moreover, the observation that multiple markers fail to provide additional information over single markers provides an impetus to focus a single diagnostic mediator in future prospective studies. Finally, the results of the current study convincingly validate our previous findings and demonstrate the promising value of sFlt-1 as a novel marker of sepsis morbidity and mortality.

\section{Limitations}

This study has a number of important limitations. First, it was a convenience sample that may have suffered from selection bias. However, the population was selected to obtain a spectrum of severities as opposed to a consecutive sample of patients. Second, we primarily only analyzed blood from the initial draw, and except in the septic shock subset, did not follow biomarkers over time. Third, in our modeling, we adjusted for age, gender, and co-morbidity, but other important confounders may have affected our results. Fourth, circulating levels of endothelial biomarkers are only indirect measures of endothelial cell activation, and thus may not accurately reflect the degree, nature and site of activation of the intact endothelium. While we have selected representative biomarkers, others may still be more accurate. Fifth, we did not include a population of non-infected critically ill patients (for example, trauma patients) so we are unable to answer whether the endothelial cell changes are specific to sepsis, or broader markers of illness severity that would extend across disease states. Finally, our sample size is reasonable, but a larger study may have afforded the opportunity for more complete subset analysis. Both our sample over time analysis and mortality analysis was limited by a small sample size.

\section{Conclusions}

The data presented here provide compelling evidence that sepsis in humans is associated with activation of the endothelium as evidence by increased levels of circulating biomarkers. We did not, however, test whether these changes were specific to sepsis, or whether endothelial cell activation occurs in critically ill patients with other insults such as trauma related inflammation; this is an important future study. Our results do support the hypothesis that the endothelium is a potential important diagnostic and therapeutic target in sepsis research.

\section{Key messages}

- There is an association between markers of endothelial cell activation/dysfunction and severity of illness and organ dysfunction in sepsis.

- There is good correlation between biomarkers associated with endothelial cell activation suggesting a net endothelial response in sepsis.

- sFLT-1 shows promise as a novel prognostic marker in sepsis.

\section{Abbreviations}

APACHE II score: acute physiologic and chronic health evaluation II score; AUC: area under the curve; BIDMC: Beth Israel Deaconess Medical Center; ED: emergency department; ICAM-1: soluble intercellular adhesion molecule; IL-6: Interleukin-6; PAI-1: plasminogen activator inhibitors -1; sFlt1: soluble fms-like tyrosine kinase-1; SIRS: systemic inflammatory response syndrome; SOFA score: sequential organ failure assessment score; VCAM-1: soluble vascular cell adhesion molecule; VEGF: vascular endothelial growth factor.

\section{Acknowledgements}

We are grateful to all local physicians, the nursing staff, research team, and patients who participated in this study. We thank Steve Moskowitz for his artwork.

Funding sources: This study was supported by an investigator initiated grant from Eli Lilly. While no investigator received direct salary support, the grant was used to pay for supplies and assays. Supplies and a device for measuring point-of-care lactate levels were provided by Abbott Point-ofCare.

Dr. Shapiro is supported in part by National Institutes of Health grants HL091757, GM076659, and 5R01HL093234-02. Dr. Schuetz was supported by a research grant from the Swiss Foundation for Grants in Biology and Medicine (Schweizerische Stiftung für medizinisch-biologische Stipendien, SSMBS). Dr. Yano is supported by National Institutes of Health grant GM088184. Dr. Parikh is supported by NIH grant 5R01HL093234-02. Dr. Jones is supported by NIH grant GM076652. Dr. Trzeciak is supported by NIH grant GM083211. Dr. Aird is supported by National Institutes of Health grants HL091757 and GM088184.

\section{Author details}

'Department of Emergency Medicine, Beth Israel Deaconess Medical Center,1 Deaconess Road CC2-W, Boston, MA 02215, USA. ${ }^{2}$ Center for Vascular Biology Research, Beth Israel Deaconess Medical Center, 99 Brookline Avenue, Boston, MA 02215, USA. ${ }^{3}$ Division of Nephrology, Beth Israel Deaconess Medical Center, 99 Brookline Street, Boston, MA 02215, USA. ${ }^{4}$ Division of General Medicine, Department of Medicine, Beth Israel Deaconess Medical Center, 1309 Beacon Street, Office 203, Brookline, MA 02446, USA. ${ }^{5}$ Department of Emergency Medicine Carolinas Medical Center, 1000 Blythe Blvd, Charlotte, NC 28203, USA. ${ }^{6}$ Cooper University Hospital, One Cooper Plaza, Camden, NJ 08103, USA. 


\section{Authors' contributions}

NS and WA conceived of the project and oversaw all components of the project and manuscript preparation. MS and KY played a substantial role in data acquisition. PS, SP, AJ, ST and LN contributed substantially to data interpretation and analysis. All authors contributed to writing the manuscript and have given final approval of the version to be published.

\section{Competing interests}

This project was funded in part by an investigator initiated research grant from Eli Lilly. Dr. Shapiro has received research grants from Hutchinson Technologies, Eli Lilly, and Inverness Medical and has received speaker's honorarium from Inverness Medical. Dr. Schuetz receives speaker's honoraria from BRAHMS's Diagnostics and Biomerieux Inc. Dr. Trzeciak receives research support from Ikaria and serves as a consultant to Spectral Diagnostics, but does not receive any personal remuneration from any commercial interest.

Received: 18 March 2010 Revised: 27 June 2010

Accepted: 13 October 2010 Published: 13 October 2010

\section{References}

1. Aird WC: The role of the endothelium in severe sepsis and multiple organ dysfunction syndrome. Blood 2003, 101:3765-3777.

2. Angus DC, Linde-Zwirble WT, Lidicker J, Clermont G, Carcillo J, Pinsky MR: Epidemiology of severe sepsis in the United States: analysis of incidence, outcome, and associated costs of care. Crit Care Med 2001, 29:1303-1310.

3. Aird WC: Spatial and temporal dynamics of the endothelium. J Thromb Haemost 2005, 3:1392-1406.

4. Shapiro NI, Yano K, Okada H, Fischer C, Howell M, Spokes KC, Ngo L, Angus DC, Aird WC: A prospective, observational study of soluble flt-1 and vascular endothelial growth factor in sepsis. Shock 2008, 29:452-457.

5. Charlson ME, Pompei P, Ales KL, Mackenzie CR: A new method of classifying prognostic comorbidity in longitudinal studies: development and validation. J Chronic Dis 1987, 40:373-383.

6. Bone R: American College of Chest Physicians/Society of Critical Care Medicine Consensus Conference: definitions for sepsis and organ failure and guidelines for the use of innovative therapies in sepsis. Crit Care Med 1992, 20:864-874.

7. Shapiro N, Howell MD, Bates DW, Angus DC, Ngo L, Talmor D: The association of sepsis syndrome and organ dysfunction with mortality in emergency department patients with suspected infection. Ann Emerg Med 2006, 48:583-590, 590 e581.

8. Vincent J, Moreno R, Takala J, Willatts S, De Mendonça A, Bruining H, Reinhart C, Suter P, Thijs L, Working Group on Sepsis-Related Problems of the European Society of Intensive Care Medicine: The SOFA (sepsis-related organ failure assessment) score to describe organ dysfunction/failure. Intensive Care Medicine 1996, 22:707-710.

9. Knaus WA, Draper EA, Wagner DP, Zimmerman JE: APACHE II: a severity of disease classification system. Crit Care Med 1985, 13:818-829.

10. Howell MD, Donnino M, Clardy P, Talmor D, Shapiro NI: Occult hypoperfusion and mortality in patients with suspected infection. Intensive Care Med 2007, 33:1892-1899.

11. Shapiro NI, Fisher C, Donnino M, Cataldo L, Tang A, Trzeciak S, Horowitz G, Wolfe RE: The feasibility and accuracy of point-of-care lactate measurement in emergency department patients with suspected infection. J Emerg Med 2010, 39:89-94.

12. Levy MM, Fink MP, Marshall JC, Abraham E, Angus D, Cook D, Cohen J, Opal SM, Vincent UL, Ramsay G: 2001 SCCM/ESICM/ACCP/ATS/SIS International Sepsis Definitions Conference. Intensive Care Med 2003, 29:530-538.

13. Moreno R, Vincent IL, Matos R, Mendonca A, Cantraine F, Thijs L, Takala J, Sprung $C$, Antonelli M, Bruining $H$, Willatts S: The use of maximum SOFA score to quantify organ dysfunction/failure in intensive care. Results of a prospective, multicentre study. Working Group on Sepsis related Problems of the ESICM. Intensive Care Med 1999, 25:686-696.

14. Janssens U, Graf C, Graf J, Radke PW, Konigs B, Koch KC, Lepper W, vom Dahl J, Hanrath P: Evaluation of the SOFA score: a single-center experience of a medical intensive care unit in 303 consecutive patients with predominantly cardiovascular disorders. Sequential Organ Failure Assessment. Intensive Care Med 2000, 26:1037-1045.
15. Balci C, Sungurtekin H, Gurses E, Sungurtekin U: [APACHE II, APACHE III, SOFA scoring systems, platelet counts and mortality in septic and nonseptic patients]. Ulus Travma Acil Cerrahi Derg 2005, 11:29-34

16. DeLong ER, DeLong DM, Clarke-Pearson DL: Comparing the areas under two or more correlated receiver operating characteristic curves: a nonparametric approach. Biometrics 1988, 44:837-845.

17. Aird WC: Phenotypic heterogeneity of the endothelium: I. Structure, function, and mechanisms. Circ Res 2007, 100:158-173.

18. Aird WC: Phenotypic heterogeneity of the endothelium: II. Representative vascular beds. Circ Res 2007, 100:174-190.

19. Springer TA: Traffic signals for lymphocyte recirculation and leukocyte emigration: the multistep paradigm. Cell 1994, 76:301-314.

20. Yano K, Liaw PC, Mullington JM, Shih SC, Okada H, Bodyak N, Kang PM, Toltl L, Belikoff B, Buras J, Simms BT, Mizgerd JP, Carmeliet P, Karumanchi SA, Aird WC: Vascular endothelial growth factor is an important determinant of sepsis morbidity and mortality. J Exp Med 2006, 203:1447-1458.

21. Shapiro NI, Yano K, Sorasaki M, Fischer C, Shih SC, Aird WC: Skin biopsies demonstrate site-specific endothelial activation in mouse models of sepsis. J Vasc Res 2009, 46:495-502.

22. Leeuwenberg JF, Smeets EF, Neefjes JJ, Shaffer MA, Cinek T, Jeunhomme TM, Ahern TJ, Buurman WA: E-selectin and intercellular adhesion molecule-1 are released by activated human endothelial cells in vitro. Immunology 1992, 77:543-549.

23. Pigott R, Dillon $L P$, Hemingway $I H$, Gearing AJ: Soluble forms of E-selectin, ICAM-1 and VCAM-1 are present in the supernatants of cytokine activated cultured endothelial cells. Biochem Biophys Res Commun 1992, 187:584-589.

24. Newman W, Beall LD, Carson CW, Hunder GG, Graben N, Randhawa ZI, Gopal TV, Wiener-Kronish J, Matthay MA: Soluble E-selectin is found in supernatants of activated endothelial cells and is elevated in the serum of patients with septic shock. J Immunol 1993, 150:644-654.

25. Dello Sbarba P, Rovida E: Transmodulation of cell surface regulatory molecules via ectodomain shedding. Biol Chem 2002, 383:69-83.

26. Kumpers P, van Meurs M, David S, Molema G, Bijzet J, Lukasz A, Biertz F, Haller $\mathrm{H}$, Zijststra JG: Time course of angiopoietin-2 release during experimental human endotoxemia and sepsis. Crit Care 2009, 13:R64.

27. Kuhns DB, Alvord WG, Gallin JI: Increased circulating cytokines, cytokine antagonists, and E-selectin after intravenous administration of endotoxin in humans. J Infect Dis 1995, 171:145-152.

28. Lemaire $L C$, de Kruif MD, Giebelen IA, van Zoelen MA, van't Veer C, van der Poll T: Differential dose-dependent effects of prednisolone on shedding of endothelial adhesion molecules during human endotoxemia. Immunol Lett 2008, 121:93-96.

29. Boldt J, Muller M, Kuhn D, Linke LC, Hempelmann G: Circulating adhesion molecules in the critically ill: a comparison between trauma and sepsis patients. Intensive Care Med 1996, 22:122-128.

30. Kayal S, Jais JP, Aguini N, Chaudiere J, Labrousse J: Elevated circulating Eselectin, intercellular adhesion molecule 1, and von Willebrand factor in patients with severe infection. Am J Respir Crit Care Med 1998, 157:776-784.

31. Cummings CJ, Sessler CN, Beall LD, Fisher BJ, Best AM, Fowler AA: Soluble E-selectin levels in sepsis and critical illness. Correlation with infection and hemodynamic dysfunction. Am J Respir Crit Care Med 1997, 156:431-437.

32. Endo $S$, Inada K, Kasai T, Takakuwa T, Yamada Y, Koike S, Wakabayashi G, Niimi M, Taniguchi S, Yoshida M: Levels of soluble adhesion molecules and cytokines in patients with septic multiple organ failure. J Inflamm 1995, 46:212-219.

33. Leone M, Boutiere B, Camoin-Jau L, Albanese J, Horschowsky N, Mege JL, Martin C, Dignat-George F: Systemic endothelial activation is greater in septic than in traumatic-hemorrhagic shock but does not correlate with endothelial activation in skin biopsies. Crit Care Med 2002, 30:808-814.

34. Sessler CN, Windsor AC, Schwartz M, Watson L, Fisher BJ, Sugerman HJ, Fowler AA: Circulating ICAM-1 is increased in septic shock. Am J Respir Crit Care Med 1995, 151:1420-1427.

35. Aird WC: Vascular bed-specific hemostasis: Role of endothelium in sepsis pathogenesis. Crit Care Med 2001, 29:S28-35.

36. Kinasewitz GT, Yan SB, Basson B, Comp P, Russell JA, Cariou A, Um SL, Utterback B, Laterre PF, Dhainaut JF: Universal changes in biomarkers of coagulation and inflammation occur in patients with severe sepsis, 
regardless of causative micro-organism (ISRCTN74215569). Crit Care 2004, 8:R82-90.

37. Lorente JA, Garcia-Frade L, Landin L, de Pablo R, Torrado C, Renes E, Garcia-Avello A: Time course of hemostatic abnormalities in sepsis and its relation to outcome. Chest 1993, 103:1536-1542.

38. Kidokoro A, Iba T, Fukunaga M, Yagi Y: Alterations in coagulation and fibrinolysis during sepsis. Shock 1996, 5:223-228.

39. Martinez MA, Pena JM, Fernandez A, Jimenez $M$, Juarez $S$, Madero $R$, Vazquez JJ: Time course and prognostic significance of hemostatic changes in sepsis: relation to tumor necrosis factor-alpha. Crit Care Med 1999, 27:1303-1308.

40. Savioli M, Cugno M, Polli F, Taccone P, Bellani G, Spanu P, Pesenti A, lapichino G, Gattinoni L: Tight glycemic control may favor fibrinolysis in patients with sepsis. Crit Care Med 2009, 37:424-431.

41. Garcia-Segarra G, Espinosa G, Tassies D, Oriola J, Aibar J, Bove A, Castro P, Reverter JC, Nicolas JM: Increased mortality in septic shock with the 4G/ 4G genotype of plasminogen activator inhibitor 1 in patients of white descent. Intensive Care Med 2007, 33:1354-1362.

42. Kim I, Moon SO, Kim SH, Kim HJ, Koh YS, Koh GY: Vascular endothelial growth factor expression of intercellular adhesion molecule 1 (ICAM-1), vascular cell adhesion molecule 1 (VCAM-1), and E- selectin through nuclear factor-kappa B activation in endothelial cells. J Biol Chem 2001, 276:7614-7620.

43. Reinders ME, Sho M, Izawa A, Wang P, Mukhopadhyay D, Koss KE, Geehan CS, Luster AD, Sayegh MH, Briscoe DM: Proinflammatory functions of vascular endothelial growth factor in alloimmunity. J Clin Invest 2003, 112:1655-1665.

44. Lucerna M, Mechtcheriakova D, Kadl A, Schabbauer G, Schafer R, Gruber F, Koshelnick Y, Muller HD, Issbrucker K, Clauss M, Binder BR, Hofer E: NAB2, a corepressor of EGR-1, inhibits vascular endothelial growth factormediated gene induction and angiogenic responses of endothelial cells. J Biol Chem 2003, 278:11433-11440.

doi:10.1186/cc9290

Cite this article as: Shapiro et al:: The association of endothelial cell signaling, severity of illness, and organ dysfunction in sepsis. Critical Care 2010 14:R182.

\section{Submit your next manuscript to BioMed Central and take full advantage of:}

- Convenient online submission

- Thorough peer review

- No space constraints or color figure charges

- Immediate publication on acceptance

- Inclusion in PubMed, CAS, Scopus and Google Scholar

- Research which is freely available for redistribution

Submit your manuscript at www.biomedcentral.com/submit 